\title{
Definição de meta comercial para crédito e projeção de passivos com modelo ARIMA
}

\section{Carlos Eduardo Bandeira}

Lorí Viali
Pontifícia Universidade Católica do Rio Grande do Sul, Especialização em

Métodos Quantitativos, Porto Alegre, RS, Brasil

E-mails: ceduardobandeira@gmail.com viali@pucrs.br

Recebido em: 13 jun. 2018. Aceito: 05 julho 2018.

DOI: http://dx.doi.org/10.21674/2448-0479.44.606-617

\section{Resumo}

O processo de definição de metas comerciais em instituições financeiras é extremamente particular. Durante seu processo de construção, cada empresa opta por uma opção que maximizará seus resultados e será condizente com sua capacidade e alcance operacionais. Neste sentido, este artigo busca resgatar o passado recente através da análise de séries temporais, valendo-se do modelo paramétrico ARIMA como ferramenta de apoio à tomada de decisão.

Palavras-chave: ARIMA. Projeção de Ativos e Passivos. Instituições Financeiras. Bancos.

\begin{abstract}
The process of setting business goals in financial institutions is extremely particular. During its construction process, each firm chooses an option that will maximize your results and be consistent with their capacity and operational range. In this sense, this article seeks to rescue the recent past through the time series analysis, taking advantage of the parametric ARIMA model as a support tool to decision.
\end{abstract}

Keywords: ARIMA. Assets and Liabilitties Projections. Financial Institutions. Banks.

\section{Introdução}

As operações de Crédito Imobiliário sãos exclusivas das instituições financeiras integrantes do Sistema Brasileiro de Poupança e Empréstimo (SBPE). No Regulamento Anexo à Resolução no 3.932/2002 do Conselho Monetário Nacional - CMN, foi estabelecido em seu artigo 1ํ, inciso I, que os recursos oriundos de depósitos de poupança devem ser aplicados em operações de financiamento imobiliário que totalizem, no mínimo, $65 \%$ (sessenta e cinco por cento) do saldo captado.

Na mesma resolução, é estabelecido que $20 \%$ do saldo de poupança deve ser aplicado em encaixe obrigatório no Banco Central (depósitos compulsórios - conforme artigo 1ํㅡㄹ inciso II); e ainda, no artigo 18, é estabelecido que todos os recursos não direcionados conforme estabelecido no artigo $1^{\circ}$ devem ser compulsoriamente recolhidos à alíquota de $20 \%$.

Nesse contexto, o saldo ideal na carteira de Crédito Imobiliário de uma instituição financeira é de $65 \%$ do volume captado em poupança, pois isso implica em menor recolhimento compulsório e maior rentabilidade dos recursos captados. Entretanto, para estabelecer uma meta comercial atrelada 
ao volume da poupança, se faz necessário um modelo de previsão adequado dos mesmos, que é o objetivo desse trabalho. Para isso, será tomada como base uma série mensal de saldos de poupança em uma instituição financeira do Rio Grande do Sul.

Como metodologia, serão utilizados testes e critérios de análise de séries temporais, buscando sempre aceitação pelo modelo que melhor explicar a série estudada. Nesse aspecto, considerando a escolha do estudo de comportamento dos dados como principal fator determinante de seu modelo preditivo, optou-se pela metodologia ARIMA.

A metodologia ARIMA, segundo Gujarati e Porter (2011), também conhecida como metodologia Box-Jenkins (BJ), tem ênfase na análise probalística, ou estocástica (análise de uma coleção de variáveis aleatórias ordenadas no tempo) das propriedades da própria série temporal, sob a filosofia deixe os dados falarem por si mesmos. Todos os modelos BJ permitem que os valores da série sejam explicados por seus valores anteriores, defasados e dos termos do erro (resíduos, ou diferenças entre os termos observados e estimados).

Inicialmente com auxílio de ferramenta computacional estatística e econométrica (Eviews 6). Apesar da sequência de testes e escolha de modelo preditivo de séries temporais ser fruto de divergência entre autores, este trabalho visa escolher o modelo que melhor explicar a série em questão e apresentar o melhor resultado preditivo. Logo, o objetivo deste trabalho não é explicar a estatística, mas sim se utilizar da mesma nas séries temporais do estudo. Todos os valores foram multiplicados por uma constante para preservar os números da empresa, sem prejuízo ao modelo preditivo. Ao final do trabalho será analisado o comparativo entre observado e realizado, demonstrando os volumes utilizados.

\section{Materiais e métodos}

\section{Verificação dos dados coletados e escolha do modelo mais adequado}

A série de depósitos de poupança observada está no intervalo de tempo de quarenta e oito observações, ou quatro anos. O objetivo do estudo é projetar o saldo para o quinto ano (12 meses). Os saldos, cujo montante mensal é na ordem de bilhões, podem facilitar o aparecimento de resíduos com valores aparentemente elevados nos testes, mas de pequena significância quando comparados ao volume total. Inicialmente, foram efetuados testes para análise da série e determinação do melhor modelo auto-regressivo (ARIMA), incluindo no estudo variáveis binárias - ou dummys - para cada mês do ano.

\section{Teste de Estacionariedade}

O objetivo do teste de estacionariedade é verificar se a série se desenvolve no tempo ao redor de uma média constante, refletindo alguma forma de equilíbrio estável que possibilite maior adequação do modelo preditivo. De acordo com Gujarati e Porter (2011), o trabalho empírico baseado nos dados de séries temporais supõe que a série temporal subjacente seja estacionária; o mesmo complementa, ainda, que uma série temporal não estacionária terá uma média que varia com o tempo, ou uma variância que varia com o tempo, ou ainda, ambas. Para verificar a estacionariedade da série, foi utilizado o teste Dickey-Fuller Aumentado, (ADF), que irá identificar também a ordem de diferenciação (d) do modelo ARIMA. 
Como a maioria dos testes estatísticos, o ADF possui uma hipótese nula e uma alternativa. Sua hipótese nula $\left(\mathrm{H}_{0}\right)$ afirma a existência de raiz unitária e, por consequência, a não estacionariedade da série temporal e necessidade de diferenciação. Sua hipótese alternativa $\left(\mathrm{H}_{1}\right)$, implica na ausência de raiz unitária e presença de estacionariedade; assim, o teste terá sucesso quando for possível aceitar hipótese alternativa, rejeitando assim a hipótese nula, em um intervalo de $95 \%$ de confiança.

\section{Primeiro teste: ADF sem diferenciações (de nível)}

Na primeira tentativa de verificação de estacionariedade, foi observado o teste ADF de nível, onde a hipótese nula é a não estacionariedade, que indicará necessidade de aplicar diferenciação de primeira ordem. A hipótese alternativa, por sua vez, é a estacionariedade. Para este teste, através do software Eviews, foi obtido o seguinte resultado:

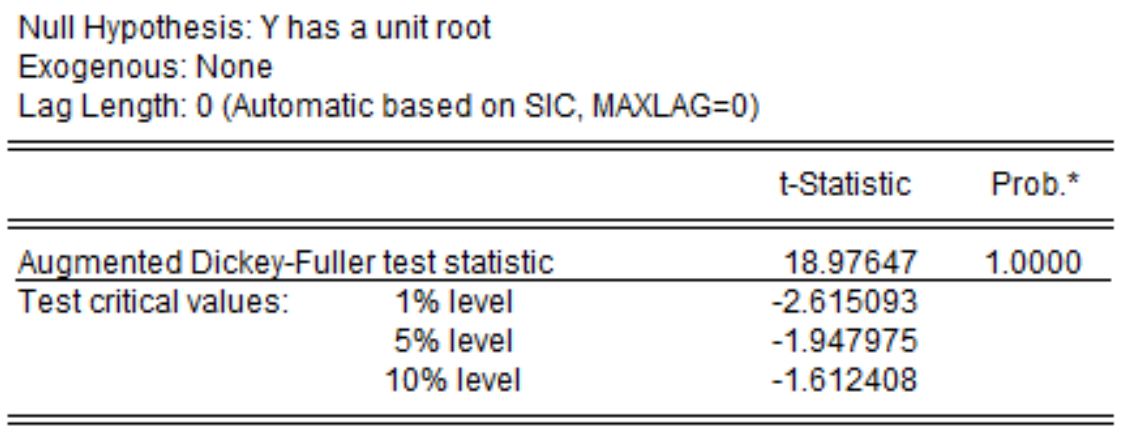

*MacKinnon (1996) one-sided p-values.

Figura 1 - Primeiro teste ADF, sem diferenciação

Conclusão do teste: é possível observar que o valor da estatística Tau ( $\mathrm{T}$ ) encontrada foi de 18,976, e o valor crítico da estatística Dickey-Fuller ao nível de $5 \%$ foi de -1,947; como t não está no intervalo - $\infty$ à $-1,947$, não é possível rejeitar a hipótese nula, uma vez que a série possui uma raiz unitária, sendo assim não estacionária.

\section{Segundo Teste: ADF com uma diferenciação}

$\mathrm{Na}$ segunda tentativa de encontrar estacionariedade da série, foi efetuado teste ADF com uma diferenciação no software Eviews, onde foi obtido o seguinte resultado: 


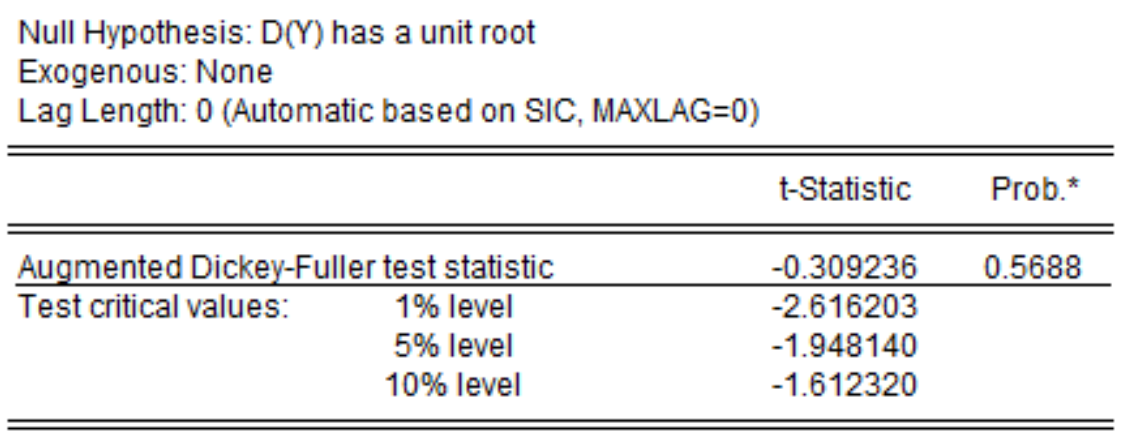

*MacKinnon (1996) one-sided p-values.

Figura 2 - Segundo teste ADF, com uma diferenciação

Conclusão do teste: o valor da estatística Tau (T) encontrada foi de -0,309, e o valor crítico da estatística Dickey-Fuller ao nível de $5 \%$ foi de -1,948; como t não está no intervalo - $\infty$ à $-1,948$, não é possível rejeitar a hipótese nula, uma vez que a série possui uma raiz unitária, sendo assim não estacionária.

\section{Terceiro teste: ADF com duas diferenciações}

$\mathrm{Na}$ terceira tentativa de encontrar estacionariedade da série, foi efetuado teste ADF com duas diferenciações no software Eviews, onde foi obtido o seguinte resultado:

\begin{tabular}{|c|c|c|}
\hline & t-Statistic & Prob.* \\
\hline Augmented Dickey-Fuller test statistic & -7.886564 & 0.0000 \\
\hline $\begin{array}{lc}\text { Test critical values: } & 1 \% \text { level } \\
& 5 \% \text { level } \\
& 10 \% \text { level }\end{array}$ & $\begin{array}{l}-2.617364 \\
-1.948313 \\
-1.612229\end{array}$ & \\
\hline
\end{tabular}

*MacKinnon (1996) one-sided p-values.

Figura 3 - Terceiro teste ADF, com duas diferenciações

Conclusão do teste: o valor da estatística Tau (т) encontrada foi de -7,886, e o valor critico da estatística Dickey-Fuller ao nível de $5 \%$ foi de-1,948; т está no intervalo $-\infty$ à $-1,948$, é possível rejeitar a hipótese nula e concluir que finalmente a série, na segunda diferenciação está estacionária. Foi identificada também a ordem (segunda) do modelo auto-regressivo que se deseja utilizar na projeção da série. 


\section{Função de Correlação (FAC), determinação da defasagem e MA}

Por meio do software Eviews, foi contruído o correlograma da série temporal de depósitos de poupança analisados, considerando as duas diferenciações já utilizadas. O objetivo aqui é a identificação, inclusive gráfica, dos termos auto-regressivos e da média móvel:

\begin{tabular}{|c|c|c|c|c|c|c|c|c|}
\hline \multicolumn{2}{|c|}{ Autocorrelation } & \multicolumn{2}{|c|}{ Partial Correlation } & & $A C$ & PAC & Q-Stat & Prob \\
\hline 1며 & 1 & 1미 & 1 & & -0.201 & -0.201 & 1.9871 & 0.159 \\
\hline & 1 & $\square$ & 1 & & -0.241 & -0.294 & 4.9118 & 0.086 \\
\hline 1 & 1 & 10 & 1 & 3 & 0.004 & -0.135 & 4.9125 & 0.178 \\
\hline 1며 & 1 & $\square$ & 1 & & -0.203 & -0.359 & 7.0875 & 0.131 \\
\hline 10 & 口 & 10 & 1 & 5 & 0.122 & -0.104 & 7.8883 & 0.162 \\
\hline 10 & 51 & 1 며 & 1 & 6 & 0.042 & -0.165 & 7.9835 & 0.239 \\
\hline 10 & p 1 & 1 & 1 & 7 & 0.111 & 0.073 & 8.6826 & 0.276 \\
\hline 10 & $p \quad 1$ & 1 & 1 & 8 & 0.063 & 0.079 & 8.9144 & 0.350 \\
\hline 10 & 1 & & 马 & 9 & -0.068 & 0.136 & 9.1890 & 0.420 \\
\hline$\square$ & 1 & $\square$ & 1 & 10 & -0.291 & -0.263 & 14.372 & 0.157 \\
\hline 10 & 1 & 1맘 & 1 & 11 & -0.080 & -0.253 & 14.780 & 0.193 \\
\hline 1 & $\square$ & 1 & $\square$ & 12 & 0.484 & 0.304 & 30.022 & 0.003 \\
\hline 1 & 1 & & ב & 13 & -0.044 & 0.102 & 30.149 & 0.004 \\
\hline 10 & 1 & 10 & 1 & 14 & -0.150 & -0.098 & 31.698 & 0.004 \\
\hline 1 & 1 & 10 & 1 & 15 & -0.017 & -0.132 & 31.719 & 0.007 \\
\hline 10 & 1 & 1 & 1 & 16 & -0.106 & 0.005 & 32.547 & 0.008 \\
\hline 1 & 1 & 11 & 1 & 17 & 0.001 & -0.074 & 32.547 & 0.013 \\
\hline 1 & 1 & 1 & 1 & 18 & 0.031 & -0.040 & 32.624 & 0.019 \\
\hline $1 f$ & 口 & 1 & 1 & 19 & 0.132 & 0.019 & 34.048 & 0.018 \\
\hline 10 & $p \quad 1$ & 1 & 1 & 20 & 0.062 & -0.072 & 34.376 & 0.024 \\
\hline 1 & 1 & 1 & 1 & 21 & -0.011 & 0.027 & 34.386 & 0.033 \\
\hline 1므 & 1 & 1 & । & 22 & -0.186 & 0.128 & 37.568 & 0.020 \\
\hline 10 & 1 & 1 & 1 & 23 & -0.103 & 0.032 & 38.587 & 0.022 \\
\hline 10 & $\square$ & 1 & 1 & 24 & 0.306 & 0.068 & 47.968 & 0.003 \\
\hline 1 & 1 & 1 & 1 & 25 & -0.016 & 0.038 & 47.996 & 0.004 \\
\hline
\end{tabular}

Figura 4 - Correlograma (visão parcial) da série com duas diferenciações

Através do Correlograma, e seguindo Gujarati e Porter (2011), foi possível observar que a variância é $1 / 48(1 / n) \cong 0,0208$, o erro padrão é de $\sqrt{0,0208}=0,1443$. O intervalo de confiança de $95 \%$ para qualquer (população) $\hat{\rho} k$ é $\hat{\rho} k \pm 1,96(0,1443)= \pm 0,2829$. Por aproximação dos valores $A C$ na Figura 1, foram efetuados testes no Eviews considerando equações com $A R_{(1)} e R_{(2)}$ (valores de AC mais próximos), verificando também a relevância de uma média móvel 2 no modelo, baseado no comportamento demonstrado pelo correlograma. Através de várias tentativas, foram testadas também as variáveis dummys (mensais) mais significativas ao modelo.

É importante ressaltar que o estudo de séries temporais através de modelos auto-regressivos está baseado somente no passado de sua própria variável para fins de previsões. Isso significa que ele não é baseado em nenhuma variável econômica e, portanto, seus coeficientes não podem ser literalmente interpretados para obtenção de resultados positivos. Assim, será examinada a plausibilidade do modelo como um todo, se este descreve bem os dados e se produz boas previsões. Após a execução de diversas composições de equação, chegou-se à melhor composição através do método ordinário dos mínimos quadrados (OLS), conforme resultado a seguir: 


\begin{tabular}{|c|c|c|c|c|}
\hline$c$ & 5984269. & 1452057. & 4.121235 & 0.0002 \\
\hline DJAN & -43111003 & 8805448. & -4.895947 & 0.0000 \\
\hline DAGO & -19931229 & 8252809. & -2.415084 & 0.0207 \\
\hline DOUT & -22242659 & 8687091. & -2.560427 & 0.0146 \\
\hline DDEZ & 32168247 & 7215491. & 4.458220 & 0.0001 \\
\hline $\operatorname{AR}(1)$ & -0.334578 & 0.159742 & -2.094489 & 0.0429 \\
\hline $\mathrm{MA}(2)$ & -0.909686 & 0.068469 & -13.28607 & 0.0000 \\
\hline R-squared & 0.720870 & \multirow{7}{*}{\multicolumn{2}{|c|}{$\begin{array}{l}\text { Mean dependent var } \\
\text { S.D. dependent var } \\
\text { Akaike info criterion } \\
\text { Schwarz criterion } \\
\text { Hannan-Quinn criter. } \\
\text { Durbin-Watson stat }\end{array}$}} & 2757035. \\
\hline Adjusted R-squared & 0.676797 & & & 22809943 \\
\hline S.E. of regression & 12967667 & & & 35.73585 \\
\hline Sum squared resid & $6.39 \mathrm{E}+15$ & & & 36.01689 \\
\hline Log likelihood & -797.0567 & & & 35.84062 \\
\hline F-statistic & 16.35623 & & & 1.854013 \\
\hline Prob(F-statistic) & 0.000000 & & & \\
\hline Inverted AR Roots & -.33 & & & \\
\hline Inverted MA Roots & .95 & -.95 & & \\
\hline
\end{tabular}

Figura 5 - Resultado do Método OLS

Nesse teste, a hipótese nula é a condição de algum dos coeficientes de regressão ser zero. A estatística t representa a relação entre eles e o erro padrão da série. Quando ele exceder 1 (um) em módulo, é provável em pelo menos $60 \%$ que o coeficiente não é zero; se ele exceder 2 (dois) em módulo, é provável em pelo menos $95 \%$ que o coeficiente não é zero. A última coluna é a de probabilidade, e representa a informação mais importante do teste, demonstrando se a hipótese nula deve ser aceita ou rejeitada. Na maioria dos casos, valores inferiores a $5 \%$ representam fortes evidências de rejeição da mesma.

Como informação adicional, temos o Critério de Informação de Akaike (AIC) e o Critério de Schwarz. Explicando de maneira breve, eles são baseados na soma dos resíduos encontrados, e concedem penalidades para grandes quantidades de variáveis e coeficientes; logo, quanto maior o valor, pior para o resultado final. Entre todos os testes efetuados, o resultado exibido na Tabela 4 representa o melhor resultado para ambos. No teste, foram examinadas também as relações das variáveis dummys mensais com o $A R_{(1)}$ e $A R_{(2)}$, bem como a média móvel 2, sempre considerando os parâmetros descritos acima. Conclusão do teste: com a rejeição da hipótese nula, aceita-se o modelo ARIMA $_{(1,2,2)}$, ou seja: com uma ordem (ou termo) auto-regressivo, duas diferenciações e dois termos da média móvel. 


\section{Análise de resíduos: teste de heterocedasticidade}

Segundo Gujarati e Porter (2011), o modelo clássico de regressão linear normal (MCRLN), que é a pedra angular da análise de séries temporais e econometria, parte de sete hipóteses. A quarta hipótese fala da homocedasticidade e a define como a variância constante do termo de erro (resíduo). Partindo dessa hipótese, foi efetuado teste de White da heterocedasticidade dos resíduos, onde temos como hipótese nula $\left(\mathrm{H}_{0}\right)$ a não heterocedasticidade (e consequente homecedasticidade), e como hipótese alternativa $\left(\mathrm{H}_{1}\right)$ a heterocedasticidade. Foi obtido o seguinte o seguinte resultado:

\begin{tabular}{lrll}
\multicolumn{4}{l}{ Heteroskedasticity Test: White } \\
\hline \hline F-statistic & 0.820727 & Prob. F(39,5) & 0.6812 \\
Obs*R-squared & 38.92029 & Prob. Chi-Square(39) & 0.4735 \\
Scaled explained SS & 23.17663 & Prob. Chi-Square(39) & 0.9791 \\
\hline \hline & & & \\
\hline \hline R-squared & 0.864895 & Mean dependent var & $1.27 \mathrm{E}+14$ \\
Adjusted R-squared & -0.188921 & S.D. dependent var & $1.71 \mathrm{E}+14$ \\
S.E. of regression & $1.86 \mathrm{E}+14$ & Akaike info criterion & 68.13234 \\
Sum squared resid & $1.73 \mathrm{E}+29$ & Schwarz criterion & 69.73827 \\
Log likelihood & -1492.978 & Hannan-Quinn criter. & 68.73102 \\
F-statistic & 0.820727 & Durbin-Watson stat & 1.972901 \\
Prob(F-statistic) & 0.681194 & & \\
\hline \hline
\end{tabular}

Figura 6 - Resultado do Teste de White

Conclusão do teste: a segunda linha da Figura 6 demonstra a estatística do teste de White. Por ser um teste de qui-quadrado, é preciso consultar os parâmetros em sua tabela de distribuição e observar, para 39 regressores (graus de liberdade), a um nível de 5\%, qual o valor crítico. Na tabela, localizamos 54,572. Como o valor demonstrado é inferior, a um nível de 5\%, aceita-se a hipótese nula, concluindo-se pela homocedasticidade dos resíduos.

\section{Análise de resíduos: teste de autocorrelação}

Outra hipótese para o modelo clássico de regressão linear normal é a não existência de autocorrelação: "Não há autocorrelação entre os termos de erro: dados quaisquer dois valores de $X$, $X_{i}$ ou $X_{j}$, (i $\left.\neq \mathrm{j}\right)$, a correlação entre quaisquer dois $u_{i}$ e $u_{j}(\mathrm{i} \neq \mathrm{j})$ é zero" (GUJARATI; PORTER, 2011, pg.88).

Para verificar a existência da autocorrelação nos resíduos, utilizaremos o teste de BreuschGodfrey (BG), utilizando o mesmo número de termos autoregressivos $(p)=1$. Nesse teste, a hipótese nula $\left(H_{0}\right)$ é a ausência de autocorrelação dos resíduos, e a hipótese alternativa $\left(H_{1}\right)$ a existência da mesma. Através desse teste foi obtido o seguinte resultado: 
Breusch-Godfrey Serial Correlation LM Test:

\begin{tabular}{lrlr}
\hline \hline F-statistic & 3.153889 & Prob. F(1,36) & 0.0842 \\
Obs*R-squared & 3.614011 & Prob. Chi-Square(1) & 0.0573 \\
\hline \hline & & & \\
\hline \hline R-squared & 0.080311 & Mean dependent var & -182036.9 \\
Adjusted R-squared & -0.124064 & S.D. dependent var & 11400168 \\
S.E. of regression & 12086673 & Akaike info criterion & 35.62996 \\
Sum squared resid & $5.26 \mathrm{E}+15$ & Schwarz criterion & 35.99129 \\
Log likelihood & -792.6741 & Hannan-Quinn criter. & 35.76466 \\
F-statistic & 0.392960 & Durbin-Watson stat & 2.018570 \\
Prob(F-statistic) & 0.917142 & & \\
\hline \hline
\end{tabular}

Figura 7 - Resultado do Teste de Breusch-Godfrey

Conclusão do teste: a segunda linha da Figura 7 demonstra a estatística do teste de BreuschGodfrey. Observando o valor crítico na tabela de distribuição qui-quadrado observamos o valor crítico de 3,841, Como o valor da probalidade é inferior, aceita-se a hipótese nula, sendo possível concluir que há ausência de autocorrelação dos resíduos.

\section{Análise de resíduos: verificação de distribuição normal}

Segundo Gujarati e Porter (2011), é condição para todo modelos clássico de regressão linear normal a presunção de normalidade na distribuição do termo de erro, ou resíduo $\left(u_{i}\right)$. Para verificar a normalidade (simetria em torno da média), foi utilizado o teste de Jarque-Bera, com a hipótese nula $\left(H_{0}\right)$ é presença de normalidade, e a hipótese alternativa $\left(H_{1}\right)$ a ausência da mesma. $O$ teste demonstrou o seguinte resultado:

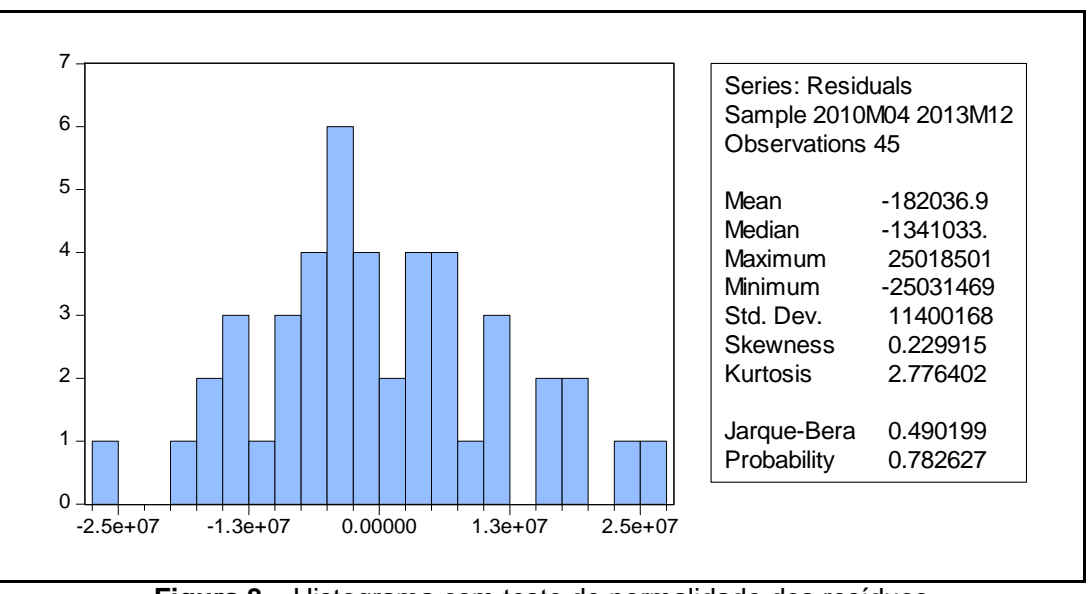

Figura 8 - Histograma com teste de normalidade dos resíduos

Conclusão do teste: a Figura 8 demonstra o coeficiente de Jarque-Bera, que é inferior a 5.99 (valor crítico de Chi-quadrado com dois graus de liberdade a um nível de 5\%). Logo, aceita-se a hipótese nula, sendo possível concluir que há distribuição normal dos resíduos, com probabilidade de $78 \%$. 


\section{Aderência e validação do modelo}

Após a realização de todos os testes em relação à série e aos resíduos, foi verificada a previsão de valores no software Eviews. Através do MAPE - Mean Absolute Percentage Error (Erro Percentual Absoluto Médio), é possível verificar a proximidade dos valores previstos e os valores reais, conforme figura abaixo:

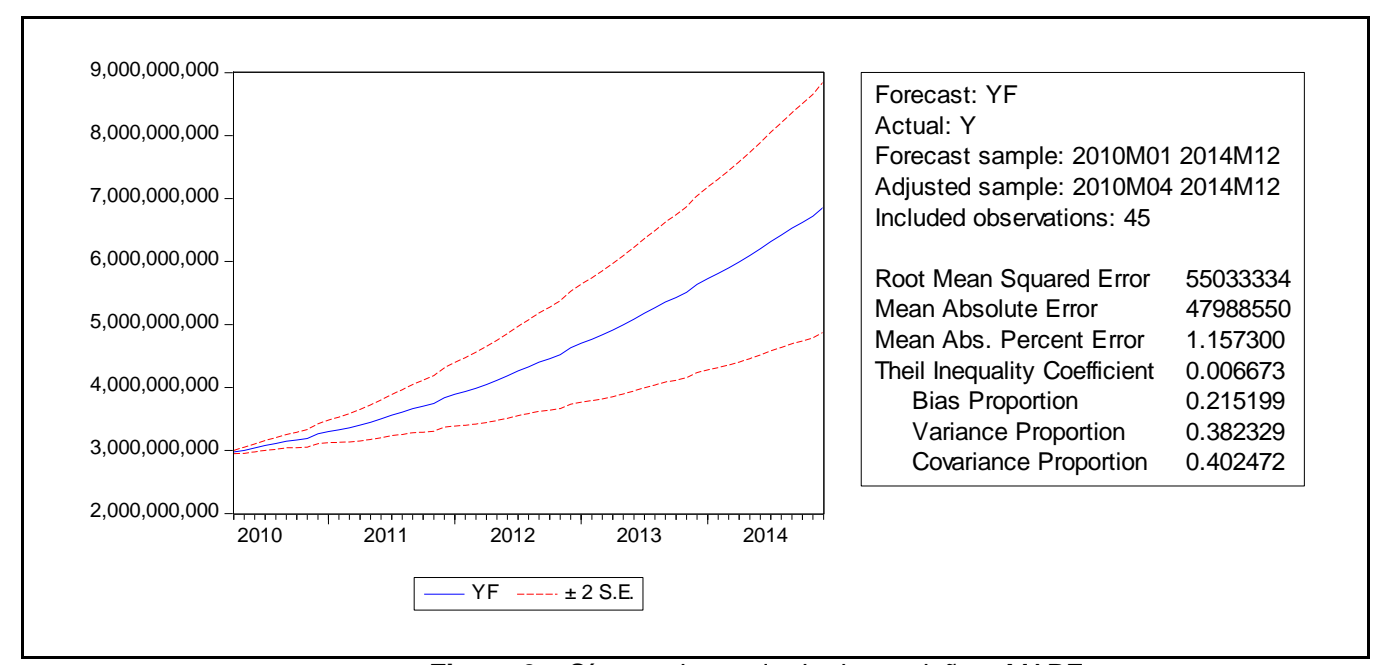

Figura 9 - Síntese do resultado de previsão e MAPE

Com o valor do erro percentual absoluto médio em 1,157 , é possível concluir que o modelo apresenta $98,84 \%(100 \%-1,157 \%)$ de proximidade com a série estudada. Outro indicador de ajuste do modelo é o coeficiente de desigualdade de Theil, aqui demonstrado por 0,006. De acordo com Soares e Castelar (2003), esse coeficiente está limitado ao intervalo [0,1], em que 0 indica um perfeito ajustamento. Após verificação do ajuste da previsão, foi efetuado comparativo do valor real com o projetado, conforme demonstrado no gráfico abaixo. Importante salientar que, mesmo exibindo a escala em milhões, as curvas praticamente se sobrepõem.

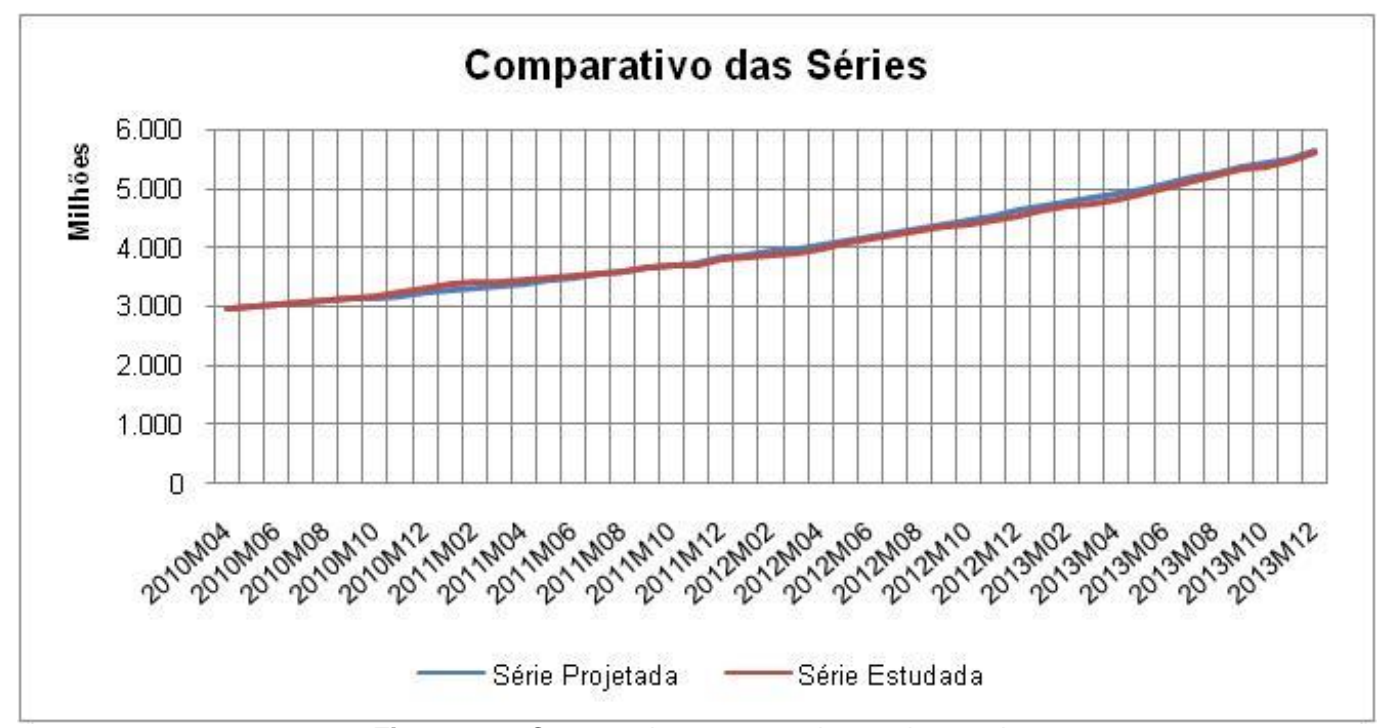

Figura 10 - Comparativo entre previsto e observado 
Como parâmetro complementar de aderência, o Eviews permite ainda a comparação gráfica da série diferenciada observada e prevista, assim como os resíduos:

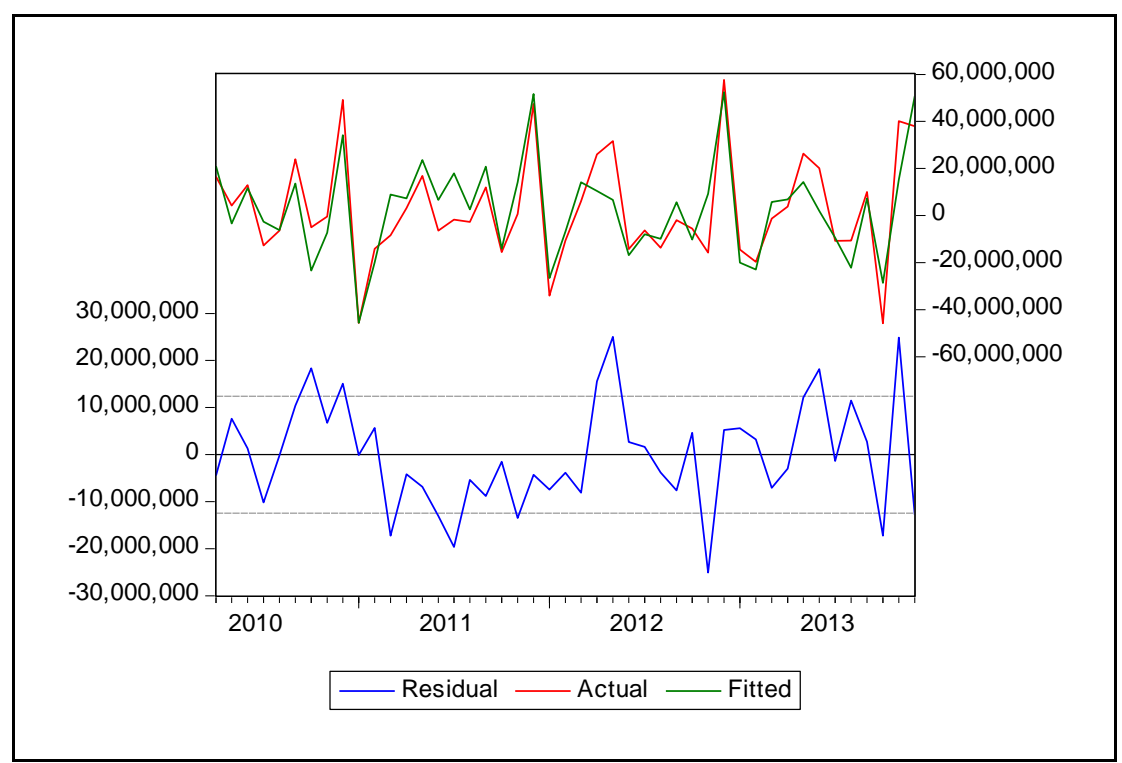

Figura 11 - Comparativo da séries diferenciadas e resíduos

\section{Aderência e validação do modelo}

Realizados todos os testes e constatada adequação do modelo ARIMA identificado, resta o cálculo da meta comercial de crédito imobiliário.

Para a análise do resultado, foi considerado o intervalo de tempo dos últimos 12 meses (considerando saldos efetivo de ambas as carteiras em questão), acrescentados de mesmo intervalo futuro, onde:

a) para a série de poupança, utilizou-se a projeção obtida do modelo ARIMA;

b) para a série de crédito imobiliário, utilizou-se o percentual da meta: $(65 \%)$.

O resultado obtido está demonstrado no gráfico a seguir:

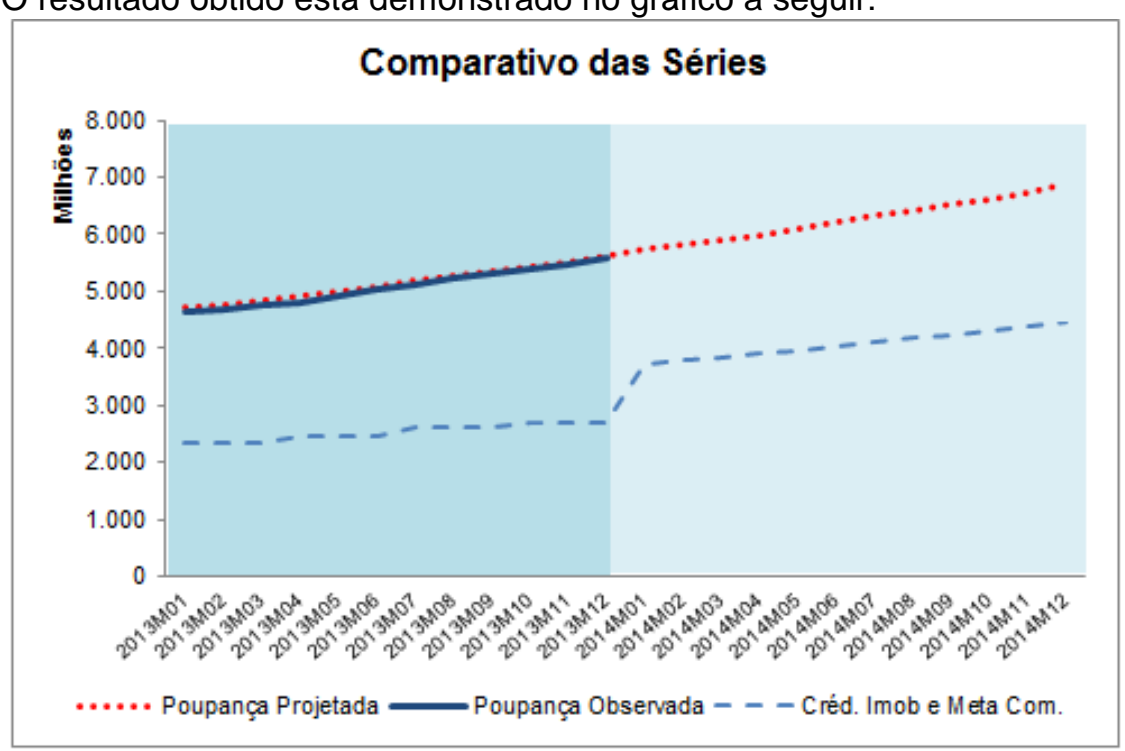

Figura 12 - Poupança projetada, observada e meta comercial 
No gráfico, o intervalo em azul mais escuro representa as séries observadas. O intervalo mais claro, a série projetada de poupança e o resultado da meta comercial.Através da análise dos dados, é possível constatar que a série de crédito comercial representava um percentual médio de $50 \%$ da poupança. Logo, a elevação da curva dessa carteira se deve ao incremento dessa relação em $15 \%$. Para efeitos comparativos, efetuamos uma projeção similar da carteira de crédito, utilizando mesma metodologia, para efeito comparativo com a meta comercial.

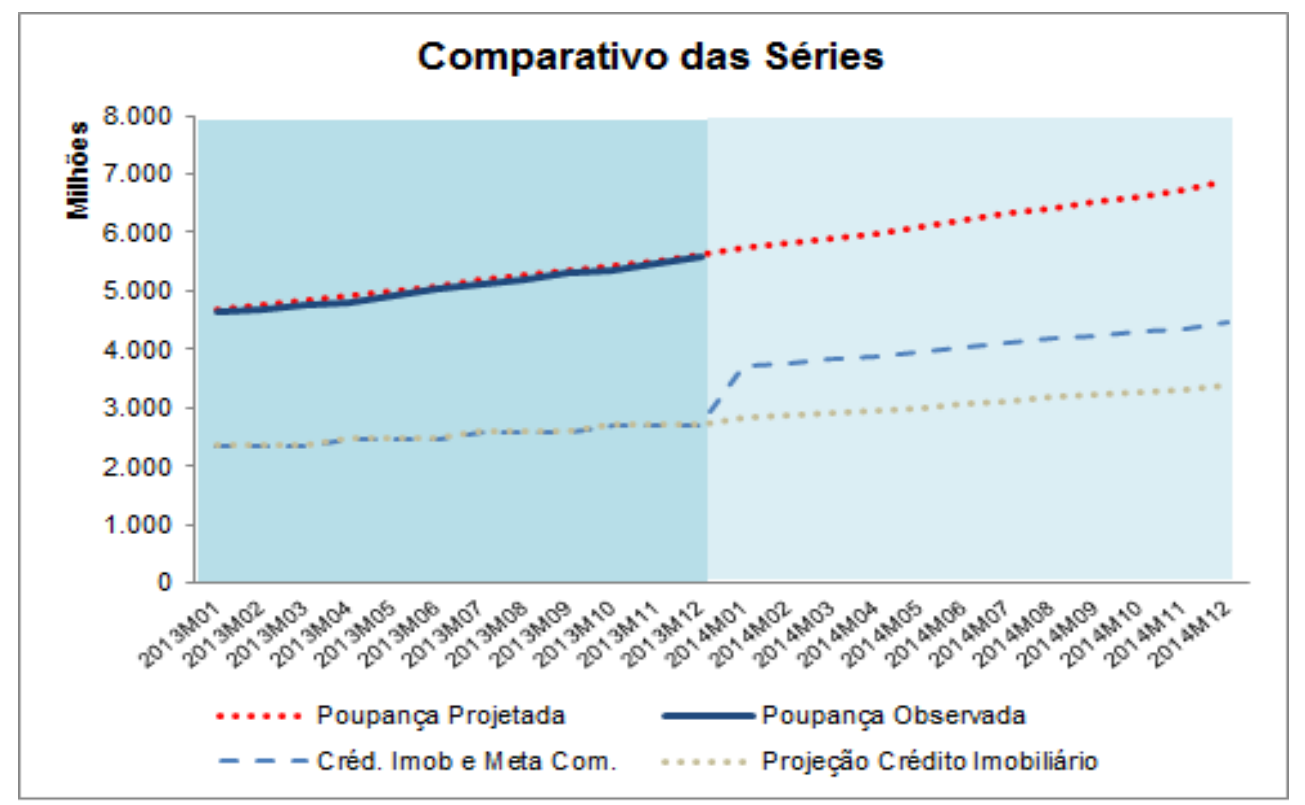

Figura 13 - Poupança projetada e observada, crédito e meta comercial

Através da Figura 13, é possível observar que a previsão de crédito imobiliário, considerando apenas sua série histórica de 48 meses, aponta para evolução da carteira em patamares inferiores à meta comercial.

\section{Considerações finais}

Com o objetivo de maximizar a rentabilidade de recursos captados na forma de depósitos de poupança através da redução de recolhimento compulsório ao Banco Central, foi estabelecida meta comercial para a carteira de crédito imobiliário.

Segundo a Resolução 4.328/2014 do CMN, a rentabilidade dos depósitos compulsórios é de aproximandamente $6,17 \%$ a.a. acrescido de TR (a mesma da poupança), enquanto a taxa média de crédito imobiliário é de $8,5 \%$ acrescida de TR; obviamente, as operações de crédito são mais rentáveis para as instituições financeiras, contribuindo com no mínimo $2,33 \%$ a.a. $(8,5 \%-6,17 \%)$ no comparativo de taxas entre captação e aplicação. Nesse sentido, não é absurdo estabelecer meta comercial mais elevada para a insituição. Entretanto, como a previsão de crédito efetuada demonstra uma evolução em níveis inferiores aos da meta, algumas ações são necessárias para estimular esse crescimento.

Uma vez que estamos falando em elevar o nível médio da curva do produto, é preciso incrementar também seu grau de atratividade no mercado. Algumas estratégias para se alcançar esse objetivo podem ser: 


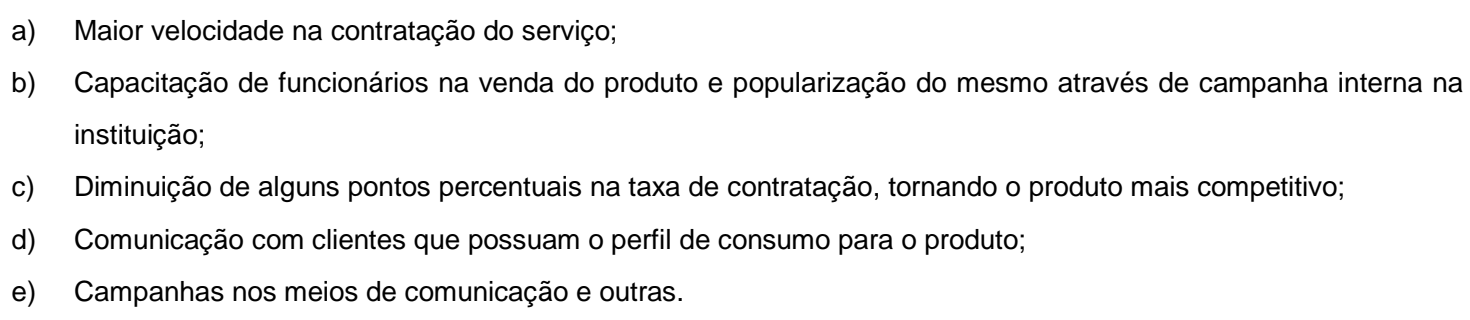

Conforme já observado na Figura 13, a previsão da série de crédito demonstra a manutenção da relação entre crédito e poupança no patamar dos $50 \%$. Caso não sejam implementadas ações diferenciadas, dificilmente a meta comercial será atingida nos próximos 12 meses.

No âmbito da análise de séries temporais, é possível concluir que sua relevância no processo foi fundamental. Antes mesmo de se utilizar das previsões que o modelo ARIMA apresenta, a análise e estudo de comportamento das séries observadas possibilita visualizar ações corretivas para se alcançar a meta desejada. Mesmo sendo uma ferramenta do processo, ela se mostrou essencial e decisiva para auxiliar a tomada de decisão.

\section{Referências}

BANCO CENTRAL DO BRASIL. Resolução 3.932/2010. Disponível em: < http://www.bcb.gov.br/pre/normativos/res/2010/pdf/res_3932_v2_P.pdf>. Acesso em: 03 nov. 2014.

BANCO CENTRAL DO BRASIL. Resolução 4.328/2014. Disponível em: < http://www.bcb.gov.br/pre/normativos/res/2014/pdf/res_4328_v1_O.pdf>. Acesso em: 03 nov. 2014.

BARROS, M. Econometria de Séries Temporais: conceitos básicos. Disponível em: < http://www.mbarros.com/documentos/upload/Capitulo\%2021\%20Gujarati\%20Resumo\%20parte\%201. pdf>. Acesso em: 03 nov. 2014.

GUJARATI, D. N.; PORTER, D. C. Econometria Básica. 5. ed. Porto Alegre: AMGH, 2011.

JOHNSON, R.R. Using Econometrics: a pratical guide. San Diego, [20--]. Disponível em: < http://www.mysmu.edu/faculty/yujun/Econ107/EViews_tutorial.pdf>. Acesso em: 03 nov. 2014.

MORETTIN, P. A.; TOLOI, C. M.C. Análise de Séries Temporais. 2 ed. São Paulo, SP: Edgard Blücher, 2006.

SOARES, I. G.; CASTELAR, I. Econometria Aplicada com o Uso do Eviews. Fortaleza, CE: Edições Livro Técnico, 2003. 